\title{
Metformin modifies the exercise training effects on risk factors for cardiovascular disease in impaired glucose tolerant adults
}

\author{
Steven K. Malin ${ }^{1}$, Joy Nightingale ${ }^{1}$, Sung-Eun Choi $^{2}$, Stuart R. Chipkin ${ }^{1}$, and Barry Braun ${ }^{1}$ \\ ${ }^{1}$ Energy Metabolism Laboratory, Department of Kinesiology, University of Massachusetts, \\ Amherst, MA 01003 \\ 2Department of Family, Nutrition, and Exercise Sciences, Queens College, Flushing, NY
}

\begin{abstract}
Impaired glucose tolerant (IGT) adults are at elevated risk for cardiovascular disease (CVD). Exercise or metformin reduce CVD risk, but the efficacy of combining treatments is unclear. To determine the effects of exercise training plus metformin, compared to each treatment alone, on CVD risk factors in IGT adults. Subjects were assigned to: placebo (P), metformin (M), exercise plus placebo (EP), or exercise plus metformin (EM) (8/group). In a double-blind design, $\mathrm{P}$ or $2000 \mathrm{mg} / \mathrm{d}$ of M were administered for 12 weeks and half performed aerobic and resistance training 3 days/week for approximately 60 minutes/day at 70\% pre-training heart rate peak. Outcomes included: adiposity, blood pressure (BP), lipids and high sensitivity C-reactive protein (hs-CRP). Z-scores were calculated to determine metabolic syndrome severity. M and EM, but not EP, decreased body weight compared to $\mathrm{P}(\mathrm{p}<0.05)$. M and EP lowered systolic BP by $6 \%$ ( $<<$ 0.05), diastolic BP by 6\% ( $<<0.05$ ), and hs-CRP by 20\% (M: trend $p=0.06$; EP: $p<0.05$ ) compared to P. Treatments raised HDL-cholesterol $(p<0.05$; EM: trend $p=0.06)$ compared to $P$ and lowered triacyglycerol $(\mathrm{p}<0.05)$ and metabolic syndrome Z-score compared to baseline (EP; trend $\mathrm{p}=0.07$ and $\mathrm{EM}$ or $\mathrm{M} ; \mathrm{p}<0.05$ ). Although exercise and/or metformin improve some CVD risk factors, only training or metformin alone lowered hs-CRP and BP. Thus, metformin may attenuate the effects of training on some CVD risk factors and metabolic syndrome severity in IGT adults.
\end{abstract}

\section{Keywords}

Prediabetes; Metabolic Syndrome; Type 2 Diabetes; Obesity; Hypertension

\section{Introduction}

Individuals with impaired glucose tolerance (IGT) are at elevated risk for cardiovascular disease (CVD) $(1,2)$ and approximately half of these individuals have metabolic syndrome (i.e. hypertensive, hyperglycemic, and dyslipidemic) (3). CVD risk is largely explained by

\footnotetext{
Users may view, print, copy, and download text and data-mine the content in such documents, for the purposes of academic research, subject always to the full Conditions of use:http://www.nature.com/authors/editorial_policies/license.html\#terms

Correspondence to: Barry Braun, PhD Dept. of Kinesiology 107 Totman Building University of Massachusetts Amherst, MA 01003 Phone: (413) 577-0146 Fax: (413) 545-2906 bbraun@kin.umass.edu.
} 
insulin resistance and excess body weight $(4,5)$. Treatments that raise insulin sensitivity or lower body weight may lower CVD risk in individuals with IGT.

The mechanisms by which exercise lowers CVD risk likely involve increasing insulin sensitivity $(6,7,8)$ and lowering circulating lipids (e.g. triacylglycerol, low-density lipoproteins, etc.), blood pressure, and C-reactive protein $(9,10,11)$. Metformin treatment can also increase insulin sensitivity $(12,13)$ and additionally, reduces fasting hyperglycemia and body weight $(14,15)$. Because the actions of exercise and metformin are potentially additive, it has been suggested that individuals with IGT and at least 1 CVD risk factor (e.g. hypertension, elevated triacylglycerol, low HDL, fasting hyperglycemia, etc.) be considered for metformin treatment while participating in a regular exercise program (16). However, the interactions between exercise and metformin on CVD risk factors have not been systematically evaluated $(17,18)$. Adding to the uncertainty, we recently showed that metformin blunted the effects of training on insulin sensitivity in men and women with IGT (19). Since insulin resistance is believed to be a key underlying factor causing metabolic syndrome (formerly syndrome $\mathrm{X}$ ), it is important to understand the effects of metformin on CVD risk after exercise training (20). Therefore, the purpose of this study was to determine the effects of combining metformin with exercise training, compared to either treatment alone, on reducing CVD risk factors in men and women with IGT. Given the opposing effects on insulin sensitivity we observed in this same study group (19), we hypothesized that the combined treatment would oppose the reduction in CVD risk compared to either treatment alone.

\section{Methods}

Overview

The effects of exercise training, metformin, or the combined treatment on cardiovascular disease (CVD) risk factors were compared in 32 otherwise healthy men and women with impaired glucose tolerance (IGT). Using a double-blind, placebo-controlled design, individuals were tested before and after 12 weeks of placebo (P), metformin (M), exercise training plus placebo (EP) or exercise training plus metformin (EM). Outcome measures included body weight, waist circumference, blood pressure, and concentrations of blood lipids, and high sensitivity C-reactive protein (hs-CRP) as well as metabolic syndrome severity.

\section{Subjects}

These subjects were the same individuals who served as the study population in our prior study on whole-body and hepatic insulin sensitivity (19). Subjects were recruited via newspaper advertisements and flyers posted in the local community. Individuals (see Table 1) were non-smoking, weight stable ( $<5 \%$ weight change over last 3 months) and free of CVD or type 2 diabetes. All subjects had impaired glucose tolerance (IGT). Individuals who were using medications to manage blood pressure $(\mathrm{M}=1 ; \mathrm{EP}=1 ; \mathrm{EM}=3)$ or cholesterol $(\mathrm{M}=1 ; \mathrm{EP}=1 ; \mathrm{EM}=1)$ were enrolled and continued treatment throughout the study. Subjects were excluded from the study if they had any contraindications to metformin use (e.g. respiratory disease, heart failure, renal/hepatic disease). All subjects were verbally 
briefed about the study and signed informed consent documents approved by the Institutional Review Board at the University of Massachusetts Amherst.

\section{Screening}

A 75 gram oral glucose tolerance test (OGTT) was used to screen individuals for IGT after a minimum 5 hour fast. Individuals with glucose concentrations between 7.8-11.1mM (140$199 \mathrm{mg} / \mathrm{dl}) 120$ minutes after consuming glucose met the criterion for IGT. Approximately half of the subjects with IGT also had impaired fasting glucose (IFG), i.e. fasting glucose concentrations between $5.5-6.9 \mathrm{mM}(100-125 \mathrm{mg} / \mathrm{dl})$. Most of the individuals in each treatment group $(\mathrm{M}=6 ; \mathrm{EP}=7$ and $\mathrm{EM}=6)$ also met ATP III criteria for metabolic syndrome (21). Only 2 participants in the $\mathrm{P}$ group had metabolic syndrome. To minimize the potential for Type- 1 and Type- 2 errors, we restricted the presentation of results to the 3 treatment groups

\section{Exercise testing}

$\mathrm{VO}_{2}$ peak was used to characterize cardiorespiratory fitness, and 1-repetition max (1-RM) tests were performed to determine strength as previously described (19).

\section{Metformin or placebo protocol}

A member of the research group, blinded to the protocol, administered pills to the subjects. All subjects were instructed to take their pills with food to minimize potential side effects. Subjects started treatment with $500 \mathrm{mg} / \mathrm{d}$ of metformin. The dose was increased $500 \mathrm{mg} / \mathrm{d}$ each week until a standard clinical dose of $2000 \mathrm{mg} / \mathrm{d}$ was reached by week 4 . This dose was maintained for the ensuing 8 weeks of the protocol.

\section{Exercise training}

Subjects underwent supervised exercise sessions 3 days/week for 60-75 minutes per session (approximately 190minutes/week total). Subjects cycled for 45 minutes at $70 \%$ of their pretraining heart rate peak 3days/week, and performed whole-body resistance exercise at $70 \%$ of the subject's 1-RM 2days/week. Subjects performed 2 sets of 12 repetitions for: chest press, latissimus pull down, leg press, bicep curl, triceps pushdown, and upright rows. Pedometers (Omron HJ112, Lake Forest, IL) were provided to all subjects and worn around the waistband for 7 consecutive days at week 0,6 and 12. These data were averaged to characterize habitual ambulation. Habitual ambulation did not change in any group after the intervention, nor were there differences across groups (data not shown).

\section{Body weight, composition \& waist circumference}

Body weight was recorded without shoes, to the nearest $0.1 \mathrm{~kg}$ on a calibrated scale, every 2 weeks at consistent times of day. Subjects were weighed in the fasted state for the first and last measurements. As previously described (19) body fat and central fat (defined as fat mass in the region bounded by the last floating rib to the top of the iliac crest/total fat mass) were assessed by Dual-energy x-ray absorptiometry (DEXA; Lunar Prodigy, Madison, WI). Waist circumference (WC) was measured to the nearest $0.25 \mathrm{~cm}$ with a plastic tape measure in the standing position approximately $2 \mathrm{~cm}$ above the umbilicus. 


\section{Food intake}

Food intake was assessed using 3-day food records at week 0, 6, and 12. Subjects selected 3 days of the week at the start of the study and the same 3 days of the week were used each time food intake was assessed. Results were averaged across the 3 days to generate a single value for energy intake at each time point. Participants were given verbal and written instructions to accurately record the types and quantities of all food and beverages consumed, including brand names and methods of food preparation. Food records were analyzed (by the same investigator S.C.) using a commercial software program (Fitday, El Segundo, California).

\section{Blood pressure, lipids and high sensitivity-CRP}

Subjects were provided isocaloric meals ( $\sim 55 \%$ carbohydrate, $30 \%$ fat, and $15 \%$ protein multiplied by 1.3 activity factor) 24 hours before baseline and week 12 measurements. Energy intake was based on resting metabolic rate measurements for 30 minutes in the supine position by indirect calorimetry (SensorMedics 800, Yorba Linda, CA). On the day of testing, subjects reported to the laboratory after an overnight fast. Systolic (SBP) and diastolic (DBP) blood pressure was determined once in the left arm using an automated system (Mark of Fitness, Inc., Shrewsbury, NJ) after at least 5-10 minutes of quiet sitting. Next, indwelling catheters were placed in a superficial vein of the forearm and fasting blood samples were collected in $3 \mathrm{~mL}$ syringes. All vacutainers were kept on ice prior to blood collection. Blood samples for the analysis of glucose were transferred to vacutainers containing sodium fluoride to inhibit glycolysis. Blood samples for the analysis of total cholesterol (TC), high-density lipoproteins (HDL), and triacylglycerol (TAG) were collected in vacutainers containing the anticoagulant EDTA. Serum samples for the analysis of hsCRP were collected in vacutainers containing a serum separator and allowed to clot for 15 minutes. Blood samples were centrifuged at room temperature for 10 minutes at 3000 RPM. Samples were immediately aliquoted and transferred to a $-80^{\circ} \mathrm{C}$ freezer until analysis.

\section{Biochemical analyses}

Fasting Plasma glucose (FPG), TC and HDL concentrations were determined enzymatically (GL5 Analyzer, Analox Instruments, Lunenberg, MA). TAG concentrations were measured by colorimetric assay (Sigma Aldrich, St. Louis, MO). Serum hs-CRP concentrations were measured using high-sensitivity ELISA kit (Diagnostic Systems Laboratory, Webster, TX). Calculations: Mean arterial pressure $(\mathrm{MAP})$ was calculated as: $\mathrm{MAP}=(2 / 3 \mathrm{DBP})+$ (1/3SBP). The cardiac risk ratio (CRR) was calculated as TC divided by HDL (19). Low density lipoprotein (LDL) was calculated using the Friedwald equation (LDL $=\mathrm{TC}-\mathrm{HDL}-$ (TAG* 0.2) (20). Metabolic syndrome Z-scores and ATP III criteria were calculated to describe the efficacy of each treatment on metabolic syndrome severity and total number of risk factors (18). Sex specific Z-scores were calculated as: male Z-score $=[(40-\mathrm{HDL}) / 10.7]$ $+[(\mathrm{TAG}-150 / 88.5)]+[(\mathrm{FPG}-100) / 11.9]+[(\mathrm{WC}-102) / 14.3]+[(\mathrm{MAP}-100) / 8.8]$, and female $\mathrm{Z}$-score $=[(50-\mathrm{HDL}) / 11.0]+[(\mathrm{TAG}-150 / 88.5)]+[(\mathrm{FPG}-100) / 11.9]+[(\mathrm{WC}-88) / 13.6]+$ [(MAP-100)/8.8]. 


\section{Statistical Analysis}

Data were analyzed using the $\mathrm{R}$ statistical software package (version 2.4.0, The $\mathrm{R}$

foundation, Vienna, Austria, 2006). Baseline subject characteristics were measured across conditions by one-way analysis of variance (ANOVA). There was no statistical difference in any baseline value except DBP. Using baseline DBP as a co-variate did not affect the response to the treatments. All other condition means were analyzed before and after the intervention using 2-way (condition by test) repeated measures ANOVA. Because baseline hs-CRP concentrations were not normally distributed, the data were log transformed for statistical analysis. When there was a significant condition $\times$ test interaction, Tukey's Post hoc analysis was used to detect differences across conditions. Paired t-tests were used to assess pre- to post differences within a condition, including caloric intake, metabolic syndrome Z-score and ATP III score. McNemar's test was used to assess metabolic syndrome prevalence (i.e. yes/no) after each treatment (22). Pearson's correlation was used to examine relationships between weight loss, cardiorespiratory fitness, CVD risk factors and insulin sensitivity (19). Significant differences were accepted as a $\leq 0.05$ and trends were reported as $0.05<a<0.10$.

\section{Results}

The raw data for fitness, body composition, fasting glucose and insulin have been published previously (19). However, given the obvious relevance of those data to risk for CVD they are summarized here for clarity.

\section{Cardiorespiratory fitness and strength}

Exercise training plus metformin (EM) and exercise training plus placebo (EP) increased $\mathrm{VO}_{2}$ peak by $10-20 \%$ compared to baseline and strength by approximately $15 \%$ compared to baseline (19).

\section{Weight change, body composition \& food intake}

Metformin (M) and EM, but not EP, decreased body weight more than $\mathrm{P}(\mathrm{p}<0.05$; Figure 1a). $\mathrm{EM}$ and $\mathrm{EP}$ also lowered percent body fat $(\mathrm{P}=-0.8 \pm 0.6 \% ; \mathrm{M}=0.1 \pm 0.5 \% ; \mathrm{EP}=-2.2$ $\pm 0.8 \% ; \mathrm{EM}=-1.5 \pm 0.7 \%)$ and central body fat $(\mathrm{P}=-0.6 \pm 0.1 \% ; \mathrm{M}=-0.1 \pm 0.6 \% ; \mathrm{EP}=$ $-1.4 \pm 0.5 \% ; \mathrm{EM}=-1.9 \pm 0.6 \%$ ) by similar amounts. Waist circumference was $2-3 \%$ lower after all 3 treatments compared to baseline (time effect: $p<0.01$; Figure 1b), but was not different across groups. Caloric intake was decreased by approximately $15 \%$ from baseline for M (2107.3 \pm 103.2 vs. $1819.8 \mathrm{kcal} ; \mathrm{p}<0.05)$ and $\mathrm{EM}(2251.0 \pm 135.7$ vs. $1777.3 \pm$ $130.2 \mathrm{kcal} ; \mathrm{p}<0.05)$. Both EP $(2292.0 \pm 177.8$ vs. $2149.2 \pm 138.1 \mathrm{kcal} ; \mathrm{p}=0.57)$ and $\mathrm{P}$ $(1923.22 \pm 135.6$ vs. $1861.3 \pm 144.5 ; \mathrm{p}=0.69)$ had no effect on intake.

\section{Fasting plasma glucose and insulin}

Exercise training and/or metformin had no effect on fasting glucose concentrations $(\mathrm{P}=-0.1$ $\pm 0.2 \mathrm{mM} ; \mathrm{M}=-0.2 \pm 0.3 \mathrm{mM} ; \mathrm{EP}=0.0 \pm 0.3 \mathrm{mM} ; \mathrm{EM}=-0.4 \pm 0.2 \mathrm{mM})$. All 3 treatments lowered fasting insulin concentrations $(\mathrm{P}=1.5 \pm 2.3 \mathrm{uU} / \mathrm{ml} ; \mathrm{M}=-5.2 \pm 1.9 \mathrm{uU} / \mathrm{ml} ; \mathrm{EP}=$ $-1.7 \pm 0.7 \mathrm{uU} / \mathrm{ml} ; \mathrm{EM}=-2.9 \pm 1.5 \mathrm{uU} / \mathrm{ml})$, but there were no group differences. 


\section{Blood pressure}

Compared to baseline, SBP and DBP increased with placebo (P; $\mathrm{p}<0.05)$. $\mathrm{M}$ and EP lowered SBP $(p<0.01)$, but all 3 treatments lowered DBP compared to $\mathrm{P}(\mathrm{p}<0.05$; Table 2). Both $M$ and EP decreased MAP compared to $P(p<0.05$; Table 2$)$ but there was no change after EM.

\section{High-sensitivity-C-reactive protein (hs-CRP)}

EP and M, but not EM, lowered CRP by approximately $20 \%$ compared to P (EP: $p<0.05$ and $\mathrm{M}$ : trend $\mathrm{p}=0.06$; Table 4).

\section{Triacylglycerol (TAG) and Cholesterol}

Compared to baseline, exercise and/or metformin lowered TAG by approximately $13 \%$ (time effect: $\mathrm{p}<0.05$; Table 3), but there were no group differences. All 3 treatments raised HDL cholesterol concentrations by $8-13 \%$ compared to $\mathrm{P}$ (M or EP; $\mathrm{p}<0.05$ and EM; trend $\mathrm{p}=0.06$; Table 3$)$. Each treatment also decreased the cardiac risk ratio $(\mathrm{p}<0.05$; Table 3$)$.

\section{Metabolic syndrome severity and prevalence}

Exercise training and/or metformin reduced the metabolic syndrome Z-score (i.e. severity) compared to baseline (EP; trend $\mathrm{p}=0.07$ and $\mathrm{EM}$ or $\mathrm{M} ; \mathrm{p}<0.05$; Figure 2a). Although each treatment reversed metabolic syndrome prevalence $(M=6$ to $1 ; \mathrm{EP}=7$ to 4 ; EM 6 to $3 ; \mathrm{p}<$ 0.05 ; Figure $2 b$ ), only EP and M reduced ATP III criteria ( $<0.05)$ compared to baseline (Figure 2c).

\section{Correlation Analysis}

Increased cardiorespiratory fitness was significantly correlated with lower fasting glucose concentrations $(r=-0.38 ; p<0.05)$ but not with any other CVD risk factor. Weight loss was correlated with lower fasting insulin concentrations $(r=0.40 ; p<0.05)$, caloric intake $(r=$ $0.46 ; \mathrm{p}<0.05)$ and waist circumference $(\mathrm{r}=0.60 ; \mathrm{p}<0.05)$. Reductions in TAG concentrations were correlated with increased insulin sensitivity $(r=-0.60 ; p<0.05)$. Lower hs-CRP concentrations were correlated with decreased systolic blood pressure $(r=$ $0.46 ; \mathrm{p}<0.05)$.

\section{Discussion}

Metformin is not only used to treat type 2 diabetes, but it is also suggested to reduce cardiovascular disease (CVD) risk factors in adults with impaired glucose tolerance (IGT) (23). Previous work suggested that exercise with metformin reduced CVD risk factors more than the respective treatments alone $(17,18)$, however, we observed no additive effect of combining exercise with metformin to reduce CVD risk factors IGT men and women. There are several possible differences between studies that may explain this. First, previous work did not systematically compare the combined treatment to either exercise (17) or metformin alone (18), thereby limiting the ability to know the effectiveness of the combined treatment on CVD risk factors. Second, recommendations to increased physical activity were not structured or supervised (18). Third, differences between HIV infected patients and IGT 
pathophysiology (17) complicate comparing previous work to ours. Last, dietary manipulations (i.e. low-fat diet) confound the ability to identify the independent effect of exercise with metformin on CVD risk factors (18). Consistent with our previous work (19), the current findings suggest that metformin may blunt some of the beneficial effects of training to lower systolic blood pressure, hs-CRP and metabolic syndrome criteria.

Weight loss (i.e. fat loss) is associated with lower CVD risk. Although exercise training with ad libitum food intake has mixed effects on weight loss $(24,25)$, combining it with metformin has been shown to cause greater weight loss $(26,27,28)$. We found that combining metformin with exercise reduced body weight by approximately $4 \mathrm{~kg}$ more than exercise alone, however, this was not different than metformin alone. Neither metformin, alone or with exercise, affected CVD risk factors (i.e. blood lipids, blood pressure, hs-CRP) more than exercise alone. Comparable reductions in central body fat between the exercise groups may explain the similar effects of exercise at reducing blood lipids (e.g. TAG and TC) (19). However, metformin decreased blood lipids equivalently to either exercise group without a change in central fat (measured by DEXA and waist circumference). Although we report reductions in waist circumferences with metformin in this study, more direct measures of central fat (e.g. MRI or CT scan) would be needed to confirm a true change in visceral fat., Despite no strong correlation between weight loss or waist circumference and CVD risk factors, we cannot rule out the possibility that fat loss explains at least some of the metformin group outcomes. In either case, these results suggest that each treatment has distinctive mechanisms for opposing CVD development.

Blood pressure is often lowered after exercise training (11), but the effects of metformin are less consistent $(13,29,30,31)$. We found that metformin yielded little added benefit to the effects of exercise training on lowering blood pressure and this outcome is consistent with other work (18). Our data suggest that the interaction of metformin with exercise training blunts reductions in systolic blood pressure, and these results may be related to changes in cardiorespiratory fitness. Although there were no statistical differences in $\mathrm{VO}_{2}$ peak between the exercise groups, the attenuated rise in $\mathrm{VO}_{2}$ peak $(\mathrm{EM}=10 \%$ vs. $\mathrm{EP}=20 \%)$ is worth considering since it is consistent with our previous work in healthy adults (32). The smaller rise in $\mathrm{VO}_{2}$ peak is likely a result of either alterations in mitochondrial adaptations or vascular blood flow. Although we did not directly measure endothelial function, we found that exercise training or metformin alone, but not the combined treatment, reduced the vascular inflammatory marker hs-CRP by approximately $20 \%$ compared to placebo. We observed a significant correlation between the change in hs-CRP and systolic blood pressure, suggesting that reduced systolic blood pressure may be related to decreased vascular inflammation. Interestingly, metformin alone reduced both systolic blood pressure and hs-CRP, but had no effect on $\mathrm{VO}_{2}$ peak compared to placebo. This result strengthens the hypothesis that there is a unique interaction between metformin and exercise on the aforementioned outcomes that could have opposing effects on cardiovascular health. From this study, we cannot determine the mechanism by which the combined treatment blunted reductions in systolic blood pressure or serum hs-CRP concentrations, but it may be related to the interaction between nitric oxide synthesis and AMPK activation $(33,34)$. Exercise combined with metformin was previously shown to blunt AMPK activation in skeletal muscle (35). Since AMPK activation is important for nitric oxide production, it is possible 
that the combined treatment blunted nitric oxide production and affected systolic blood pressure and hs-CRP concentrations.

Although combining metformin with lifestyle modification lowered TAG concentrations more than lifestyle modification alone in overweight insulin resistant adolescents $(26,28)$, there was no additive effect in IGT adults (18). Consistent with Andreadis et al (18), we found that exercise training and/or metformin had similar effects at lowering TAG concentrations, despite exercise increasing $\mathrm{VO}_{2}$ peak and reducing central body fat. The decline in TAG concentrations across treatments was associated with increased insulin sensitivity $(r=-0.60 ; p<0.05)$. Although we cannot prove causality or directionality from this correlation, a possibility is that reductions in circulating TAG lead to improved peripheral glucose uptake (36). Fasting non-esterified fatty acids (NEFA) concentrations were elevated after exercise training with metformin, which could potentially explain the blunted rise in insulin sensitivity (19). The incongruent finding between lower TAG and higher NEFA concentrations with exercise plus metformin may relate to decreased NEFA uptake in skeletal muscle and liver $(37,38)$ or increased lipolytic activity (39). We are not able to discern which of the mechanisms account for elevated NEFA concentrations, but our results support the beneficial effects of exercise to lower TAG and increase insulin sensitivity (40). Whether exercise and/or metformin treatments have a direct impact on reducing CVD incidence in individuals with IGT remains to be seen.

Approximately 50\% of individuals with IGT have metabolic syndrome (3). Lifestyle modification or metformin decrease metabolic syndrome prevalence (3), and the combined treatment may have additive effects (18). In this study, we showed that structured exercise training and/or metformin had similar effects on reducing metabolic syndrome severity (i.e. Z-score; Figure 2a). The initial ATP III score was lower in the exercise with metformin group, compared with either treatment alone. The lower baseline may have made it more difficult to detect a significant improvement from baseline, and, only exercise training or metformin alone reduced the number of subjects meeting ATP III criteria (Figure 2c). In addition, although not statistically different, metformin decreased metabolic syndrome prevalence more than the combined treatment (Figure $2 b$ ). Since the combined treatment did not lower systolic blood pressure, an ATP III criteria factor, our findings suggest that the interaction of metformin and exercise may oppose the reversal of metabolic syndrome in IGT adults compared to metformin alone. We recognize that further work is needed given the modest sample size in this study and our inability to compare treatment groups to placebo because only 2 subjects in that group had metabolic syndrome. However, the novel observation from this study is that combining exercise with metformin may have opposing effects on cardiovascular health, which could be clinically significant (16).

In conclusion, we observed that combining metformin with structured exercise training did not have additive effects on any CVD risk factor measured in this group of IGT adults. Metformin may blunt some of the effects of exercise training on lowering systolic blood pressure and hs-C-reactive protein and consequently contribute to preventing a decrease in the number of metabolic syndrome risk factors. If our results are reproduced and generalizable to men and women with IGT then the clinical utility of recommending the combination of exercise and metformin for people with IGT may need to be re-evaluated. 
Despite metformin alone reducing CVD risk factors comparably to exercise alone, the improvements in cardiorespiratory fitness after exercise is one of the best predictors of CVD and early mortality. As a result, our findings highlight the overall positive effects of exercise on lowering CVD risk in adults with IGT.

\section{Acknowledgments}

S.K.M, S.R.C, and B.B. contributed to the study design and data collection. S.K.M was primarily responsible for data analysis and statistical integrity. J.N. contributed to data entry and data analysis. S.C. was solely responsible for food data analysis. S.K.M, S.R.C, and B.B. wrote the manuscript and J.N and S.C. reviewed/edited the manuscript. The authors would like to thank Kirsten Granados, Richard Viskochil, and Robert Gerber for technical assistance and helpful discussion. B.B. receives consulting fees from Pfizer Global Inc. We thank all the dedicated participants and research assistants for assistance on this project. This research was supported by NIH 5 R56 DK081038 Grant.

\section{REFERENCES}

(1). Blake D, Meigs J, Muller D, Najjar S, Andres R, Nathan D. Impaired glucose tolerance, but not impaired fasting glucose, is associated with increased levels of coronary heart disease risk factors: results from the Baltimore Longitudinal Study on Aging. Diabetes. 2004; 53(8):20952100. [PubMed: 15277391]

(2). Festa A, D'Agostino R, Howard G, Mykknen L, Tracy RP, Haffner SM. Chronic subclinical inflammation as part of the insulin resistance syndrome: the Insulin Resistance Atherosclerosis Study (IRAS). Circulation. 2000; 102(1):42-47. [PubMed: 10880413]

(3). Orchard TJ, Temprosa M, Goldberg R, Haffner S, Ratner R, Marcovina S, et al. The effect of metformin and intensive lifestyle intervention on the metabolic syndrome: the Diabetes Prevention Program randomized trial. Ann Intern Med. 2005; 142(8):611-619. [PubMed: 15838067]

(4). Haffner S. Insulin resistance, inflammation, and the prediabetic state. Am J Cardiol. 2003; 92(4A): $18 \mathrm{~J}-26 \mathrm{~J}$.

(5). Unwin N, Shaw J, Zimmet P, Alberti KGMM. Impaired glucose tolerance and impaired fasting glycaemia: the current status on definition and intervention. Diabetic Med. 2002; 19(9):708-723. [PubMed: 12207806]

(6). Houmard JA, Tanner CJ, Slentz CA, Duscha BD, McCartney JS, Kraus WE. Effect of the volume and intensity of exercise training on insulin sensitivity. J Appl Physiol. 2004; 96(1):101. [PubMed: 12972442]

(7). Dela F, Larsen JJ, Mikines KJ, Ploug T, Petersen LN, Galbo H. Insulin-stimulated muscle glucose clearance in patients with NIDDM. Effects of one-legged physical training. Diabetes. 1995; 44(9):1010-1020. [PubMed: 7657022]

(8). Holten MK, Zacho M, Gaster M, Juel C, Wojtaszewski JFP, Dela F. Strength training increases insulin-mediated glucose uptake, GLUT4 content, and insulin signaling in skeletal muscle in patients with type 2 diabetes. Diabetes. 2004; 53(2):294-305. [PubMed: 14747278]

(9). Durstine JL, Grandjean P, Cox C, Thompson P. Lipids, lipoproteins, and exercise. J Cardiopulm Rehabil. 2002; 22(6):385-398. [PubMed: 12464825]

(10). Herder C, Peltonen M, Koenig W, Stfels K, Lindstrm J, Martin S, et al. Anti-inflammatory effect of lifestyle changes in the Finnish Diabetes Prevention Study. Diabetologia. 2009; 52(3):433442. [PubMed: 19130041]

(11). Bacon S, Sherwood A, Hinderliter A, Blumenthal J. Effects of exercise, diet and weight loss on high blood pressure. Sports Med. 2004; 34(5):307-316. [PubMed: 15107009]

(12). Musi N, Hirshman MF, Nygren J, Svanfeldt M, Bavenholm P, Rooyackers O, et al. Metformin increases AMP-activated protein kinase activity in skeletal muscle of subjects with type 2 diabetes. Diabetes. 2002; 51(7):2074-2081. [PubMed: 12086935] 
(13). Lehtovirta M, Forsén B, Gullström M, Häggblom M, Eriksson JG, Taskinen MR, et al. Metabolic effects of metformin in patients with impaired glucose tolerance. Diabet Med. 2001; 18(7):578583. [PubMed: 11553189]

(14). Cusi K, Consoli A, DeFronzo RA. Metabolic effects of metformin on glucose and lactate metabolism in noninsulin-dependent diabetes mellitus. J Clin Endocrinol Metab. 1996; 81(11): 4059-4067. [PubMed: 8923861]

(15). DeFronzo RA, Barzilai N, Simonson DC. Mechanism of metformin action in obese and lean noninsulin-dependent diabetic subjects. J Clin Endocrinol Metab. 1991; 73(6):1294-1301. [PubMed: 1955512]

(16). Rhee M, Herrick K, Ziemer D, Vaccarino V, Weintraub W, Narayan KMV, et al. Many Americans have pre-diabetes and should be considered for metformin therapy. Diabetes Care. 2010; 33(1):49-54. [PubMed: 19808929]

(17). Driscoll SD, Meininger GE, Lareau MT, Dolan SE, Killilea KM, Hadigan CM, et al. Effects of exercise training and metformin on body composition and cardiovascular indices in HIV-infected patients. AIDS. 2004; 18(3):465. [PubMed: 15090799]

(18). Andreadis EA, Katsanou PM, Georgiopoulos DX, Tsourous GI, Yfanti GK, Gouveri ET, et al. The effect of metformin on the incidence of type 2 diabetes mellitus and cardiovascular disease risk factors in overweight and obese subjects--the Carmos study. Exp Clin Endocrinol Diabetes. 2009; 117(4):175-180. [PubMed: 19053032]

(19). Malin S, Gerber R, Chipkin S, et al. Independent and Combined Effects of Exercise Training and Metformin on Insulin Sensitivity in Individuals With Prediabetes. Diabetes Care. 2011; 35(1):16.

(20). Reaven GM. Pathophysiology of insulin resistance in human disease. Physiol Rev. 1995; 1995:473-486. [PubMed: 7624391]

(21). Johnson J, Slentz C, Houmard J, Samsa G, Duscha B, Aiken L, et al. Exercise training amount and intensity effects on metabolic syndrome (from Studies of a Targeted Risk Reduction Intervention through Defined Exercise). Am J Cardiol. 2007; 100(12):1759. [PubMed: 18082522]

(22). Fleiss, JL. Statistical methods for rates and proportions. John Wiley; New York: 1981. p. 114

(23). Bailey C. Metformin: effects on micro and macrovascular complications in type 2 diabetes. Cardiovascular drugs and therapy. 2008; 22(3):215-224. [PubMed: 18288595]

(24). Church T, Martin C, Thompson A, Earnest C, Mikus C, Blair S. Changes in weight, waist circumference and compensatory responses with different doses of exercise among sedentary, overweight postmenopausal women. PloS one. 2009; 4(2):e4515-e4515. [PubMed: 19223984]

(25). Donnelly J, Hill J, Jacobsen D, Potteiger J, Sullivan D, Johnson S, et al. Effects of a 16-month randomized controlled exercise trial on body weight and composition in young, overweight men and women: the Midwest Exercise Trial. Arch Intern Med. 2003; 163(11):1343-1350. [PubMed: 12796071]

(26). Clarson C, Mahmud F, Baker J, Clark H, McKay W, Schauteet V, et al. Metformin in combination with structured lifestyle intervention improved body mass index in obese adolescents, but did not improve insulin resistance. Endocrine. 2009; 36(1):141-146. [PubMed: 19387874]

(27). Love-Osborne K, Sheeder J, Zeitler P. Addition of metformin to a lifestyle modification program in adolescents with insulin resistance. J Pediatr. 2008; 152(6):817-822. [PubMed: 18492523]

(28). Atabek M, Pirgon O. Use of metformin in obese adolescents with hyperinsulinemia: a 6-month, randomized, double-blind, placebo-controlled clinical trial. J Pediatr Endocrinol Metab. 2008; 21(4):339-348. [PubMed: 18556965]

(29). Landin K, Tengborn L, Smith U. Treating insulin resistance in hypertension with metformin reduces both blood pressure and metabolic risk factors. J Intern Med. 1991; 229(2):181-187. [PubMed: 1900072]

(30). Giugliano D, De Rosa N, Di Maro G, Marfella R, Acampora R, Buoninconti R, et al. Metformin improves glucose, lipid metabolism, and reduces blood pressure in hypertensive, obese women. Diabetes Care. 1993; 16(10):1387-1390. [PubMed: 8269798] 
(31). Dorella M, Giusto M, Da Tos V, Campagnolo M, Palatini P, Rossi G, et al. Improvement of insulin sensitivity by metformin treatment does not lower blood pressure of nonobese insulinresistant hypertensive patients with normal glucose tolerance. J Clin Endocrinol Metab. 1996; 81(4):1568-1574. [PubMed: 8636369]

(32). Braun B, Eze P, Stephens BR, Hagobian TA, Sharoff CG, Chipkin SR, et al. Impact of metformin on peak aerobic capacity. Appl Physiol Nutr Metab. 2008; 33(1):61-67. [PubMed: 18347654]

(33). Verma S, Wang C, Li S, Dumont A, Fedak PWM, Badiwala M, et al. A self-fulfilling prophecy: C-reactive protein attenuates nitric oxide production and inhibits angiogenesis. Circulation. 2002; 106(8):913-919. [PubMed: 12186793]

(34). Lira V, Brown D, Lira A, Kavazis A, Soltow Q, Zeanah E, et al. Nitric oxide and AMPK cooperatively regulate PGC-1 in skeletal muscle cells. J Physiol (Lond ). 2010; 588(Pt 18):3551. [PubMed: 20643772]

(35). Sharoff C, Hagobian T, Malin S, Chipkin S, Yu H, Hirshman M, et al. Combining short-term metformin treatment and one bout of exercise does not increase insulin action in insulin resistant individuals. Am J Physiol Endocrinol Metab. 2010; 298(4):E815-823. [PubMed: 20071560]

(36). Kraus W, Slentz C. Exercise training, lipid regulation, and insulin action: a tangled web of cause and effect. Obesity. 2009; 17(Suppl 3):S21-S26. [PubMed: 19927141]

(37). Smith AC, Mullen KL, Junkin KA, Nickerson J, Chabowski A, Bonen A, et al. Metformin and exercise reduce muscle FAT/CD36 and lipid accumulation and blunt the progression of high-fat diet-induced hyperglycemia. Am J Physiol Endocrinol Metab. 2007; 293(1):E172-181.

[PubMed: 17374701]

(38). Iozzo P, Hallsten K, Oikonen V, Virtanen K, Parkkola R, Kemppainen J, et al. Effects of metformin and rosiglitazone monotherapy on insulin-mediated hepatic glucose uptake and their relation to visceral fat in type 2 diabetes. Diabetes Care. 2003; 26(7):2069-2074. [PubMed: 12832315]

(39). Koh H, Hirshman M, He H, Li Y, Manabe Y, Balschi J, et al. Adrenaline is a critical mediator of acute exercise-induced AMP-activated protein kinase activation in adipocytes. Biochem J. 2007; 403(3):473-481. [PubMed: 17253964]

(40). Slentz C, Houmard J, Johnson J, Bateman L, Tanner C, McCartney J, et al. Inactivity, exercise training and detraining, and plasma lipoproteins. STRRIDE: a randomized, controlled study of exercise intensity and amount. J Appl Physiol. 2007; 103(2):432-442. [PubMed: 17395756] 


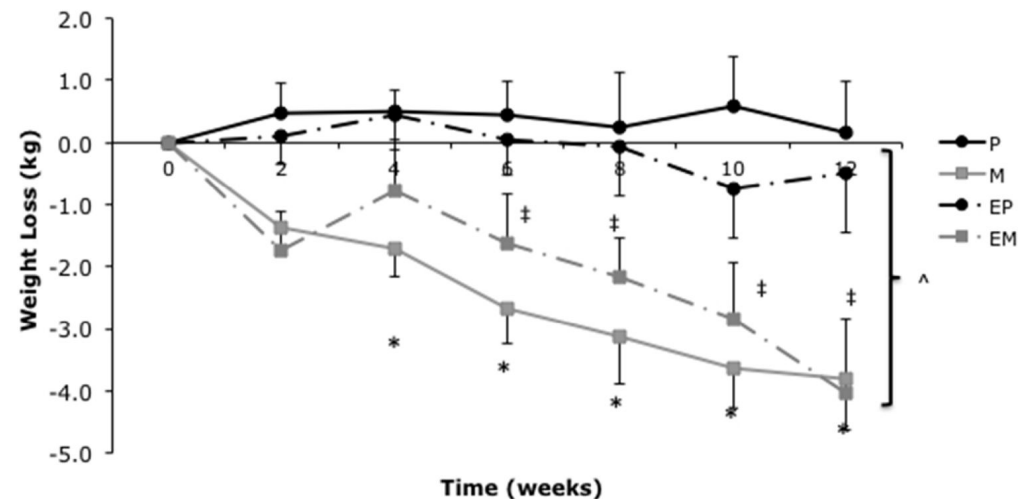

Figure 1a.

Change in body weight over the 12 week protocol. ^ Significant effect of M and EM compared to $\mathrm{P}(\mathrm{p}<0.05)$. Significant effect of $\mathrm{M}(*)$ and $\mathrm{EM}(\ddagger)$ compared to baseline ( $\mathrm{p}<$ $0.05)$. Data are mean \pm standard error of the mean. 


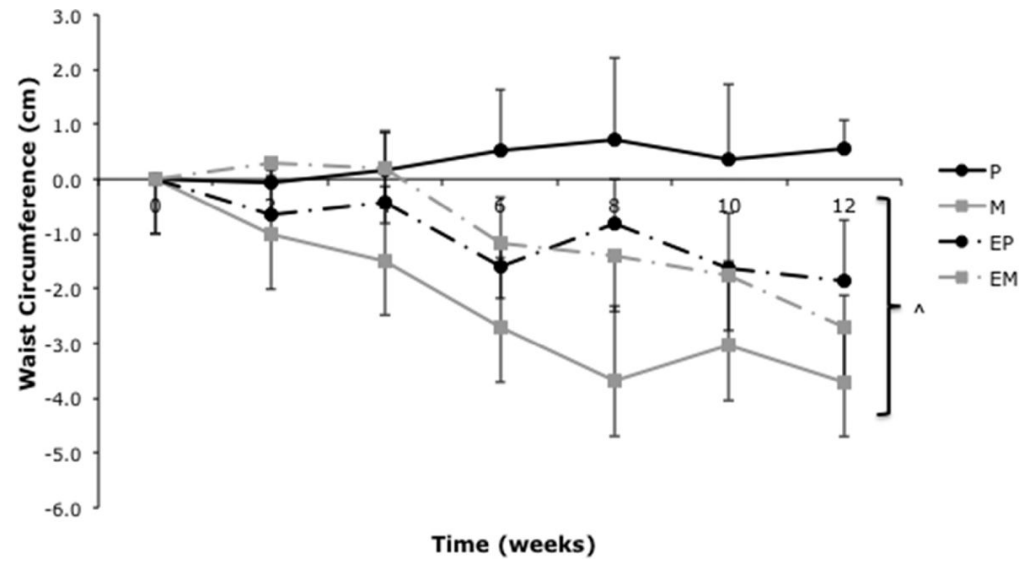

Figure 1b.

Change in waist circumference over the 12 week intervention. *Significant effect of time (p $<0.05$ ). Data are mean \pm standard error of the mean. 


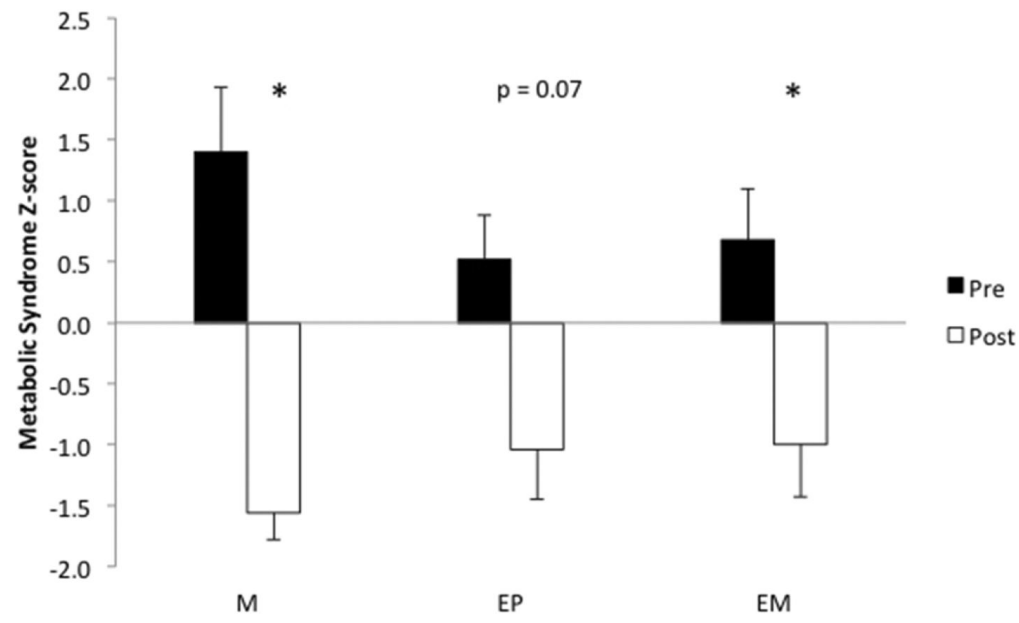

Figure 2a.

Metabolic syndrome severity before and after the 12 week intervention. *Pre to post was statistically different by paired t-test; $\mathrm{p}<0.05 .{ }^{\$} \mathrm{p}=0.07$. Data are mean \pm standard error of the mean. 


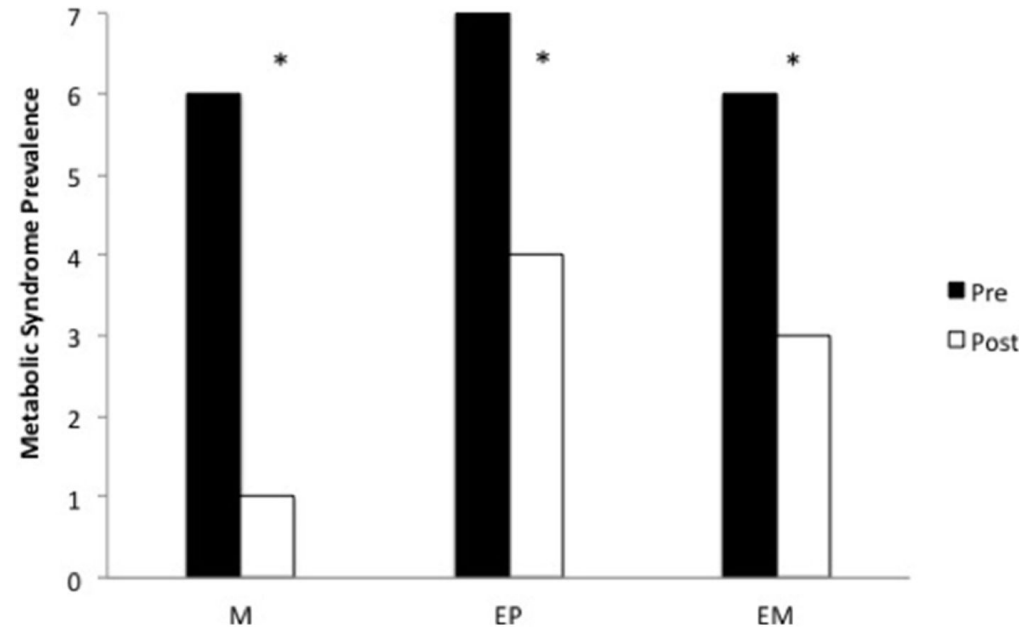

Figure 2b.

Metabolic syndrome prevalence before and after the 12 week intervention. Prevalence refers to the fraction of individuals with metabolic syndrome pre and post. *Pre to post was statistically different by McNemar; $p<0.05$. Data are frequency. 


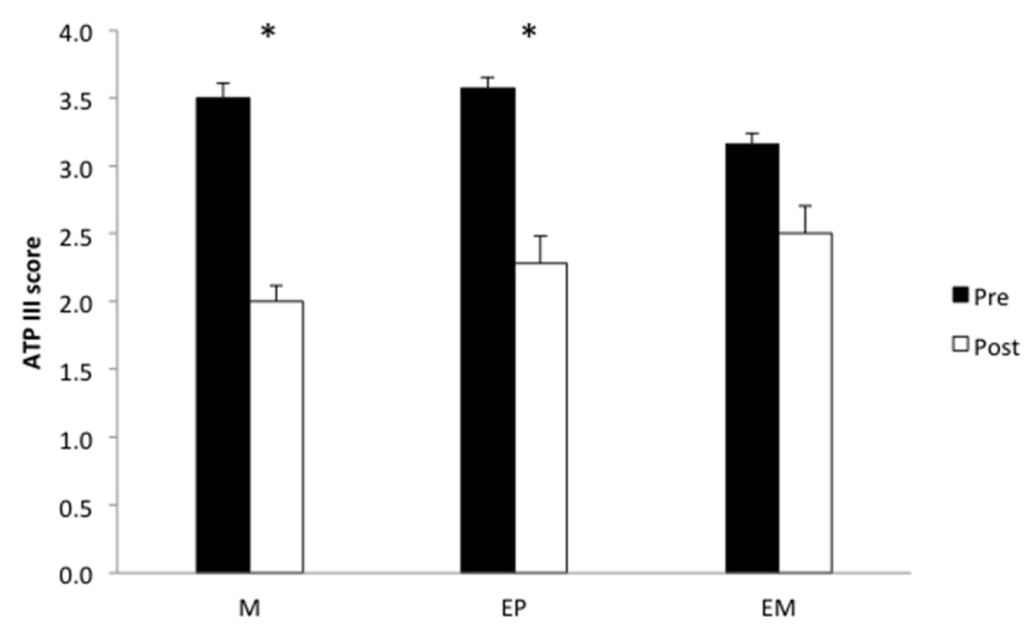

Figure 2c.

ATP III score before and after the 12 week intervention. *Pre to post was statistically different by paired t-test; $\mathrm{p}<0.05$. Data are mean \pm standard error of the mean. 
Table 1

Subject Characteristics.

\begin{tabular}{|l|c|c|c|c|}
\hline & P & M & EP & EM \\
\hline Sex & $6 \mathrm{~F} / 2 \mathrm{M}$ & $4 \mathrm{~F} / 4 \mathrm{M}$ & $5 \mathrm{~F} / 3 \mathrm{M}$ & $5 \mathrm{~F} / 3 \mathrm{M}$ \\
\hline Age $($ year) & $49.8 \pm 10.9$ & $45.0 \pm 7.5$ & $45.4 \pm 8.0$ & $49.1 \pm 6.6$ \\
\hline BMI $\left(\mathrm{kg} / \mathrm{m}^{2}\right)$ & $34.0 \pm 6.3$ & $33.9 \pm 5.2$ & $33.5 \pm 4.1$ & $31.2 \pm 5.3$ \\
\hline Body weight $(\mathrm{kg})$ & $93.5 \pm 6.0$ & $101.5 \pm 5.8$ & $95.5 \pm 5.1$ & $94.1 \pm 6.5$ \\
\hline $\mathrm{VO}_{2}$ peak $(\mathrm{ml} / \mathrm{kg}-\mathrm{ffm} / \mathrm{min})$ & $41.2 \pm 8.2$ & $43.4 \pm 10.3$ & $45.7 \pm 9.4$ & $48.2 \pm 4.9$ \\
\hline Fasting Glucose $(\mathrm{mM})$ & $5.79 \pm 0.57$ & $5.58 \pm 0.61$ & $5.42 \pm 0.42$ & $5.46 \pm 0.80$ \\
\hline 2-hour Glucose $(\mathrm{mM})$ & $9.38 \pm 1.33$ & $9.28 \pm 1.53$ & $10.23 \pm 1.02$ & $9.46 \pm 1.70$ \\
\hline Steps per day & $4983.9 \pm 2104.9$ & $5028.1 \pm 2047.7$ & $5871.7 \pm 1912.8$ & $6444.6 \pm 1529.9$ \\
\hline
\end{tabular}

No significant differences were observed between conditions.

Data are mean \pm standard deviation. 


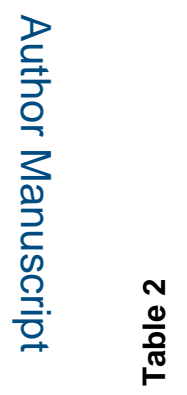

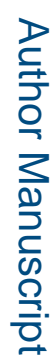

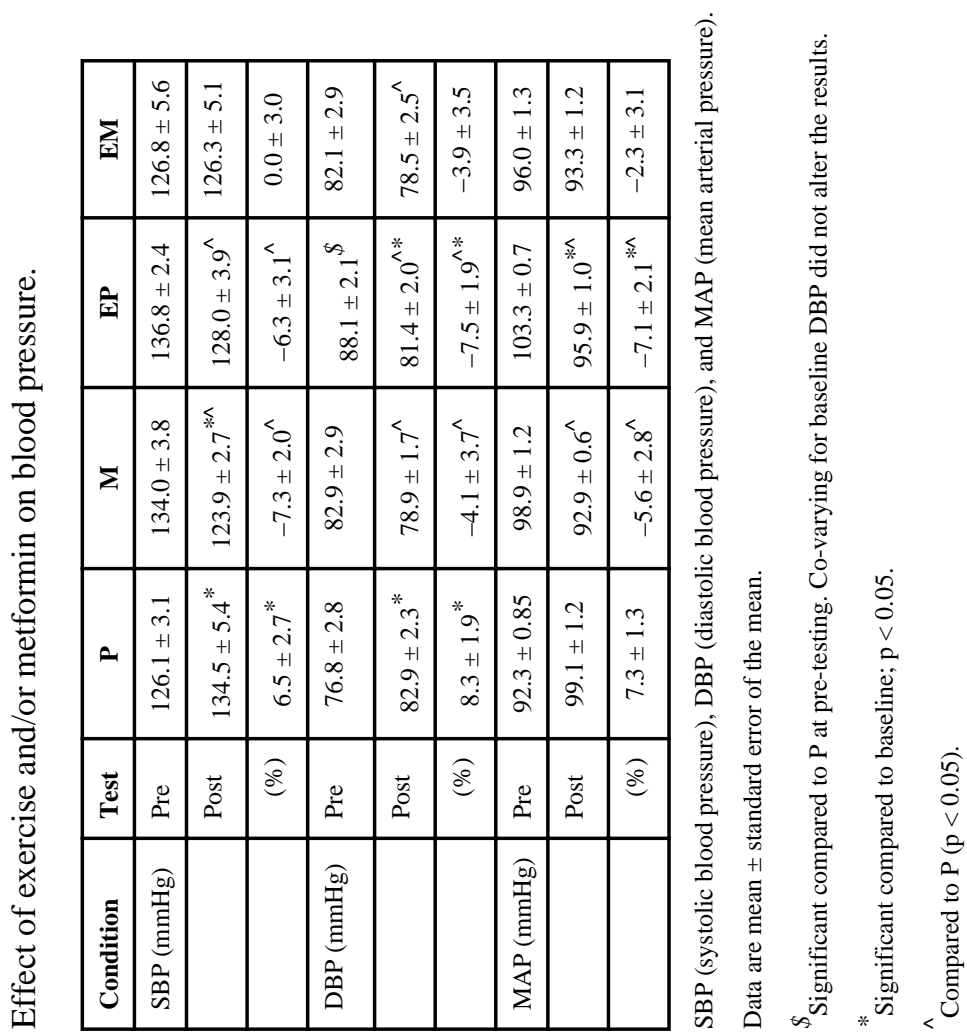

Obesity (Silver Spring). Author manuscript; available in PMC 2013 November 05. 
